Zarankiewicz Natalia, Kosz Katarzyna, Kuchnicka Aleksandra, Zielińska Martyna, Wolanin Natalia, Cisel Bogumiła. Endometriosis and ovarian cancer - what they have in common? Journal of Education, Health and Sport. 2020;10(8):230-239. eISSN 2391-8306. DOI http://dx.doi.org/10.12775/JEHS.2020.10.08.026

https://apcz.umk.pl/czasopisma/index.php/JEHS/article/view/JEHS.2020.10.08.026 https://zenodo.org/record/3987505

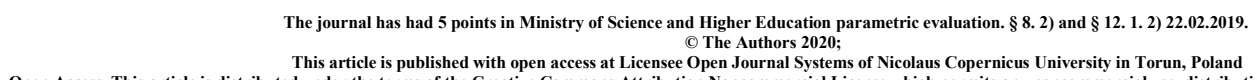

This article is published with open access at Licensee Open Journal Systems of Nicolaus Copernicus University in Torun, Poland

Open Access. This article is distributed under the terms of the Creative Commons Attribution Noncommercial License which permits any noncommercial use, distribution, and reproduction in any medium,

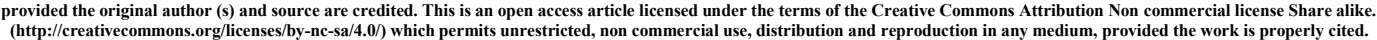
The authors declare that there is no conflict of interests regarding the publication of this paper.

Received: 01.08.2020. Revised: 05.08.2020. Accepted: 17.08.2020.

\title{
Endometriosis and ovarian cancer - what they have in common?
}

\author{
Natalia Zarankiewicz, Katarzyna Kosz, Aleksandra Kuchnicka, Martyna Zielińska, \\ Natalia Wolanin, Bogumiła Ciseł
}

Natalia Zarankiewicz

ORCID iD https://orcid.org/0000-0001-9203-3376

Affiliation $\quad$ Student Scientific Group of Oncological Surgery, Radziwiłlowska 13 (SPSK Nr 1) 20-080

Lublin

Medical University of Lublin

Country Poland

Bio Statement -

Principal contact for editorial correspondence.

Katarzyna Kosz

ORCID iD https://orcid.org/0000-0001-6848-0598

Affiliation Student Scientific Group of Oncological Surgery, Radziwillowska 13 (SPSK Nr 1) 20-080

Lublin

Medical University of Lublin

Country Poland

Bio Statement -

Aleksandra Kuchnicka

ORCID iD https://orcid.org/0000-0001-8481-7592

Affiliation Student Scientific Group of Oncological Surgery, Radziwiłlowska 13 (SPSK Nr 1) 20-080

Lublin

Medical University of Lublin

Country Poland

Bio Statement - 


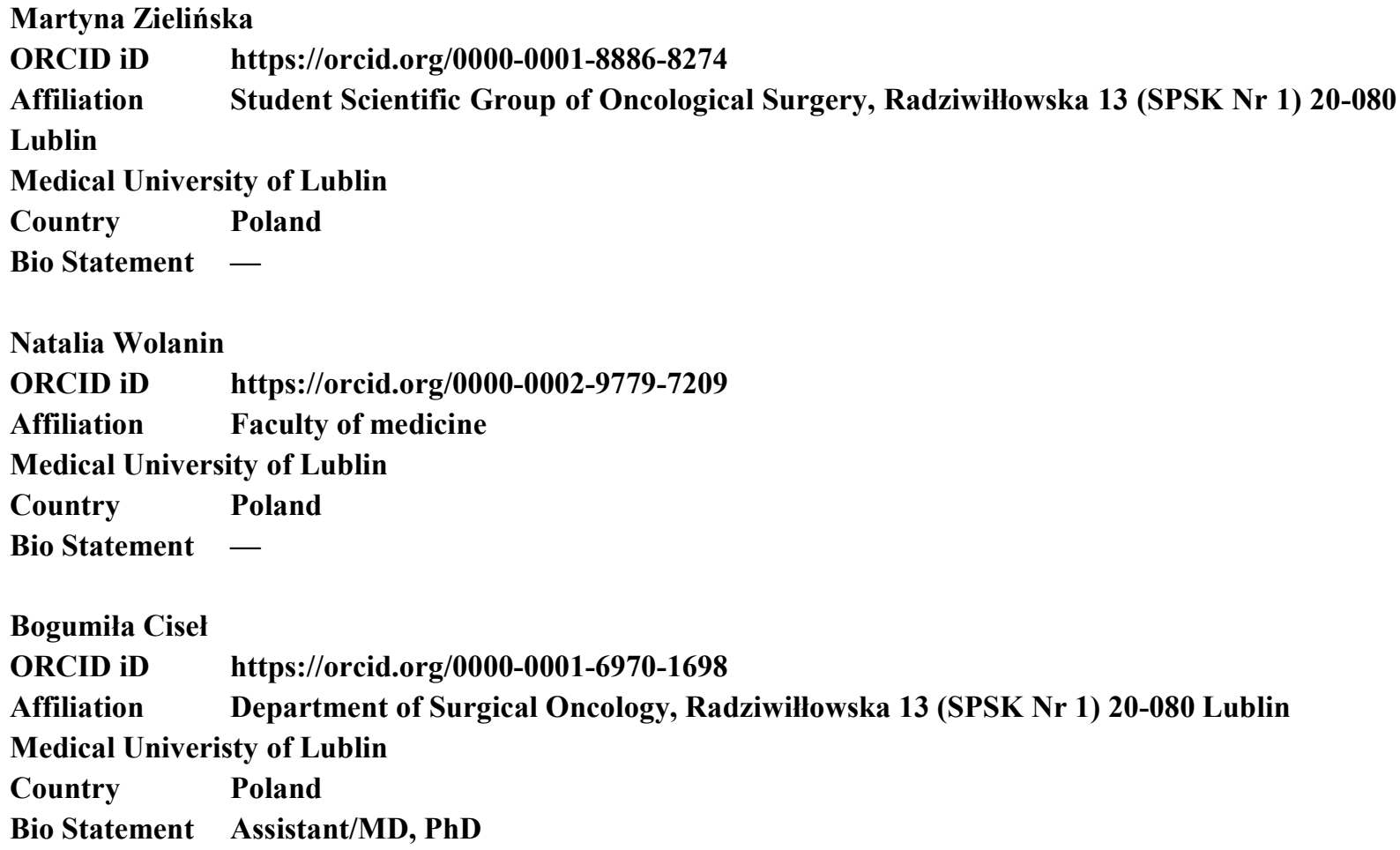

Abstract

Introduction and purpose:

Endometriosis is one of the most common diseases among women in the reproductive age. Although its etiology remains not fully understood, several models of endometriosis development are taken into consideration. Many studies have been focused on hormonal and genetic alterations in endometriosis. The results of these studies allow for a thesis on common origin of endometriosis and a few types of ovarian cancer.

The aim of this review is to present actual knowledge about endometriosis' development conditions and a possible association with ovarian cancer.

\section{Material and method:}

The review concerned articles published in years 2012-2020, which were collected in the PubMed and Google Scholar. Particular attention was paid to the development of both the endometriosis and Endometriosis-Related Ovarian Neoplasms (ERONs) and their genetic disorders.

\section{Results:}

Endometriosis development is connected with several risk factors and also immune, hormonal and genetic alterations. Some authors postulated that malignant transformation of endometriosis may be a multi-step process powered by increasing levels of estrogen. Many studies confirmed that mutations of $A R I D I A$ and PIK3CA genes are likely to be present in the majority of cases of ovarian cancer with adjacent endometriosis.

\section{Conclusions:}

The awareness of clinical features and medical complications of endometriosis is spreading increasingly around the world. Many studies are focused on genetic causes of this illness. It may be supposed that we will be able to select women with endometriosis and increased risk of ovarian cancer and prevent its development. 
Key words: endometriosis, ovarian cancer, ERONs

\section{Description of the state of knowledge Introduction and purpose}

Endometriosis is a valid problem among premenopausal women. It affects up to 10$15 \%$ of this population [1]. It is defined as the presence of endometrial glandular epithelium or stromal cells outside the uterus [2]. Endometriosis is considered to be a risk factor or even the precursor lesion for a few types of ovarian cancer. Nevertheless, only atypical endometriosis is confirmed as a direct cancer precursor. Atypical endometriosis is rare and is observed in only $1-3 \%$ of all the cases [2,3]. Ovarian cancers are divided into five histological groups: clear-cell, endometrioid, mucinous, high-grade serous and low-grade serous. The risk of developing ovarian cancer varies according to the subtype [4].

The etiology of endometriosis is still unclear. Interestingly, some of the risk factors of endometriosis are similar for ovarian cancer. These risk factors include: low parity, early menarche and short, regular periods [1]. Numerous studies were carried out to discover genetic predisposition to endometriosis due to the higher prevalence of endometriosis among close relatives. Some analyses focused on sex steroid hormone pathways and several singlenucleotide polymorphisms (SNPs) were found out. Other studies focused on somatic mutations that may lead to the development of endometriosis. Furthermore, examination of ovarian endometriosis co-occurring with ovarian cancer showed that many mutations are the same in both pathologies [2]. Loss of heterozygosity (LOH) and loss-of-function mutations in $A R I D I A$ and activating mutations of PIK3CA are the most common mutations found in endometriosis with adjacent ovarian cancer [5]. The creation of genomic-based classification of endometriosis would raise a possibility of evaluation of the risk of neoplastic transformation. Potentially, it may give a rise to the basis of the prevention [2].

The aim of this review is to present actual knowledge about the association between endometriosis and ovarian cancer. The analysis was prepared using internet resources PubMed and Google Scholar. When preparing the review, the analysis concerned publications that included key words such as: endometriosis, endometriosis-related ovarian neoplasms, genetic mutations in endometriosis and cancer, endometrioid ovarian cancer

\section{Endometriosis - a disease of unknown origin}

Endometriosis is a progressive disease and one of the most common gynecological problems in women in reproductive age. The clinical symptoms of endometriosis differ according to the patients. In the face of many clinical pictures and various endometriosis locations, making a diagnosis is still a valid problem for healthcare professionals. The most common locations for ectopic endometrial epithelium are ovaries, the ovarian fossa, the uterosacral ligaments and the pouch of Douglas [6]. One of the main symptoms of endometriosis is chronic pelvic pain. Endometriosis is present in approximately a half of women with this ailment. The next one is infertility and roughly $30 \%$ of infertile women are diagnosed with endometriosis $[2,6]$.

The etiology of endometriosis remains unclear. There are many theories about endometriosis origin. However, according to the current knowledge, four models are considered to be the most plausible. The most common is retrograde menstruation. 
It means simultaneous reflux of cells from endometrial cavity to the pelvis, through the fallopian tubes during normal menstruation. Besides, scientists are also considering the following theories: coelomic metaplasia, endometriosis induction by hematopoietic cells and embryonic cell rests theory. Coelomic metaplasia refers to the common origin of ovary and the Müllerian ducts (progenitors of the endometrium) from the coelomic epithelium. This theory seems to be reasonable in reference to endometriosis without the menstruation. Embryonic cell rests theory is rather speculative. Stem cell theory is based on a model of hematopoietic cells differentiation into endometriotic tissue. It is still an area of active research [7]. Endometriosis is divided into three anatomical subtypes: superficial peritoneal endometriosis, ovarian endometriosis and deep infiltrating endometriosis. Infiltrating form of ovarian endometriosis is associated with subsequent creation of endometriosis cysts (endometriomas). They are filled with menstrual fluid and named as a chocolate ovarian cysts $[2]$.

\section{Risk factors, immune and hormonal abnormalities}

Although the etiology of endometriosis remains unclear, its risk factors are wellknown and scientifically validated. The most important are: early menarche, short length of a menstrual cycle, low body mass index and low parity. Current studies suggest the association between low birth weight and endometriosis in later life. Moreover, prenatal exposure to a synthetic estrogen (diethylostilbestrol) is also associated with potentially increased risk of the disease [8]. It is worth mentioning the significance of estrogen in the pathogenesis of endometriosis. First of all, the blood level of estrogen in premenopausal women is significantly higher in comparison to postmenopausal women. This fact may explain why endometriotic lesions regress after menopause. Secondly, an increased expression of aromatase, which is involved in estrogen biosynthesis, was found in endometriotic lesions. It enables the local production of estradiol [9]. In addition, a decreased expression of 17bhydroxysteroid dehydrogenase- 2 was also found in endometriotic lesions. It is related to the decreased conversion of estradiol to estriol, which is the less active hormone. An important role in the pathogenesis of endometriosis is assigned to the failure of natural immunological mechanisms. In the reference to the estrogen disorders, it was reported that locally produced estradiol escalates local inflammation with an increase in prostaglandin E2 levels. It favors a development of endometriotic lesions [2].

Dysfunction of immune response in endometriosis involves abnormalities both in the activity and cells' levels. The increased level of the T-regulatory lymphocytes was reported in endometriotic lesions and peritoneal fluid among women with this disease [10,11]. Higher level of T-helper-17 (Th17) cells in peritoneal fluid was also described. Interestingly, the cells' frequency correlates with the endometriosis severity [12]. Notwithstanding, the most important immune factor affecting the endometriosis development is elevated level of peritoneal macrophages, mostly M2 subtype. M2 macrophages are responsible for tissue remodeling and angiogenesis promotion. It should be noted that besides the greater concentration of cells, peritoneal macrophages have got increased activity. They enhance lesions establishment through the production of pro-inflammatory cytokines such as: TNF- $\alpha$, IL-6 and IL-1 $\beta[2,11,13]$. 
It is worth mentioning that IL-6 is considered to be one of the most important cytokines that have been found elevated in both the peritoneal fluid and blood in women with endometriosis. On the other hand, IL-4 and IL-10 - classified as anti-inflammatory cytokines - are also upregulated in endometriotic lesions. The activity of peritoneal cytotoxic T-cells and NK cells is reduced and disturbed in endometriotic lesions. The activity of NK cells is also decreased in peripheral blood. The next important disorder in endometriosis is polyclonal B cells activation and intensified autoantibodies production. Although the presence of autoantibodies could suggest an autoimmune background, further studies are required to develop this viewpoint $[1,11,14]$. The study described by Porpora et al. compared the prevalence of some autoimmune diseases among women with and without endometriosis. The study focused on diseases such as: systemic lupus erythematosus (SLE), celiac disease (CD), inflammatory bowel disease (IBD) and autoimmune thyroiditis. The study involved 298 women: 148 women affected by endometriosis in the case group and 150 women without this disease in the control group. There were eight SLE cases $(5,4 \%)$ in the case group and only one patient in the control group ( $\mathrm{OR}=8.63 ; 95 \% \mathrm{CI} 1.07-69.91 ; \mathrm{p}=0.01)$. Five patients were diagnosed with IBD $(3,4 \%)$ in the case group and there was not a single case of IBD in the control group $(\mathrm{p}=0.07)$. The number of $\mathrm{CD}$ cases was as follows: fifteen in the case group $(10,2 \%)$ and only one in the control group $(0,7 \%)(\mathrm{OR}=12.04 ; 95 \% \mathrm{CI} 2.22-130.79$; $\mathrm{p}<0.0001)$. There were 80 cases of thyroiditis in the case group $(54,4 \%)$ and fourteen controls $(9,3 \%) \quad(\mathrm{OR}=11.37 ; 95 \% \mathrm{CI} \quad 6-21.5 ; \mathrm{p}<0.0001)$. This significant association between endometriosis and other autoimmune diseases supports the assumption of autoimmune background of endometriosis [15].

\section{Genetics of endometriosis}

On the basis of the increased prevalence of endometriosis among relatives, many studies have attempted to explain genetic predisposition to this disease. Nonetheless, it should be clearly stated that endometriosis is a multifactorial condition, involving also some somatic mutations.

Several studies have focused on genetic pathways related to the sex steroid hormones. Sapkota et al. found significant single-nucleotide polymorphisms among 17045 endometriosis cases evaluated in large meta-analysis. Including minimal and mild endometriosis, the most substantial associations with endometriosis were confirmed in GREB1, WNT4, KDR, ID4 genes and in the intergenic region at 7p15.2. SNP in WNT4 (1p36.12) is considered to be one of the most significant in endometriosis. This polymorphism is also commonly observed in ovarian cancer. Besides, study indicated novel loci associated with endometriosis. They were found in FN1, CCDC170, SYNE1, FSHB genes and in region located at $7 \mathrm{p} 12.3$. These SNPs seem to be more frequent in moderate-tosevere stages of endometriosis [16].

Actual data provides evidence for the association between endometriosis and a dysregulation of microRNAs (miRNAs), which are regarded as a possible biomarkers in endometriosis. Some studies reported downregulation of miR-17-5, miR-20a and increase in miR-125a and miR-222 levels in ovarian endometriosis. These miRNAs are involved in angiogenesis regulation. Moreover, impaired expression of miR-9 was also reported and this phenomenon is considered to be related to increased levels of apoptotic Bcl-2 protein. 
This finding may be a clue to understanding the changed levels of apoptotic proteins in endometriotic tissue. The miRNAs may be utilized as therapeutic targets in the future [17].

\section{Endometriosis and ovarian cancer - do they have common features?}

Ovarian tumors associated with endometriosis are commonly known as Endometriosis-Related Ovarian Neoplasms (ERONs) [18]. Besides ovarian cancer, several other tumors were also observed in women with endometriosis, including endometrial stromal sarcoma [19], non-Hodgkin's lymphoma and brain tumors [1]. Endometrioid, clear cell and low-grade serous subtypes of ovarian cancer are considered to be the most frequently observed ovarian cancers with adjacent endometriosis [20]. The association between ovarian cancer and endometriosis varies according to the authors. Some analyses describe endometriosis as a risk factor for ovarian cancer [1]. The standpoint that atypical endometriosis is a direct precursor for ovarian cancer becomes generally accepted nowadays [21]. Atypical endometriosis is characterized by architectural proliferation and increase in nuclear/cytoplasmic ratio [2]. Neoplastic transformation seems to be a multi-step process, where endometriotic lesions accumulate sufficient amounts of mutations to become neoplasms themselves [5].

In the case of endometrioid ovarian cancer, it is worth drawing attention to synchronous type I endometrial cancer and endometriosis-related ovarian endometrioid tumor. With reference to retrograde menstruation model, two scenarios for the origin of endometrioid cancers were proposed. It is not fully explained whether endometrioid ovarian cancer is caused by reflux and migration of normal or initially cancerous endometrial cells. First option assumes that secondarily to normal retrograde menstruation, endometrial cells implant the ovary. It comes to the development of inflammation, iron-associated oxidative stress and dysfunction of hormonal balance. These processes drive to the malignant transformation. Second model explains the origin of simultaneous endometrial and ovarian cancers as the result of metastatic dissemination between endometrial tissue and ovary. This model was finally chosen to be more frequent [3]. A considerable argument for the common origin of endometrial and ovarian cancer was introduced in the study conducted by Mangili et al. in 2012. It was alleged that among 65 patients, the rate of occurrence of endometrial cancer was higher in women with concurrent endometriosis-related ovarian cancer than in women without ovarian pathology $(33 \%$ vs. $11 \%, \mathrm{p}=0.04)$. Moreover, the histological concordance between these cancers achieved the value of $92 \%(p=0.004)$ [22]. It gives a rise to the hypothesis that cancerous endometrial cells migrate to the pelvis - via the menstrual reflux- and go through the transformation into the atypical endometriosis and then finally become endometrioid ovarian cancer [3].

Many studies provide scientific evidences for common origin for both the endometriosis and ovarian cancer. Firstly, ovarian cancers and endometriosis share some characteristic features, including: dysregulation of apoptosis, progressive and estrogendependent growth, neovascularization, the ability to implant distant tissues and creation of microenvironment which enables disease development, for example through the dysfunction of immune response [1,6]. Secondly, genomic instability in endometriosis and ovarian cancers seems to be related with similar mutations. 
Genetic mutations located on chromosome $1 \mathrm{p} 36$ are considered to be valid factors in the transition from endometriosis to cancer. These genes are ARIDIA and CDH5, which belong to the tumor suppressor genes [23]. Interestingly, the loss-of-function mutation of $A R I D 1 A$ is the most common mutation observed in atypical endometriosis with contiguous ovarian cancer [18]. Activating mutations of the PIK3CA gene are also commonly found in ovarian cancers with linked atypical endometriosis [5]. Furthermore, the malignant transformation of endometriosis is related with loss of heterozygosity at 10q23.3 and mutations in other tumor suppressor genes- PTEN and TP53. Inactivating mutation of PTEN and loss-of-function of ARIDIA are considered as early steps in carcinogenesis of endometriotic lesions. Mutations of TP53 are related with endometriosis adjacent to ovarian cancer, but they were not observed in benign endometriotic lesions without cancer. Newer studies suggest that mutations in TP53 seem to be related mostly with high-grade endometrioid ovarian cancer [24]. In the study described by Anglesio et al. seven cases of clear cell ovarian cancer with synchronous endometriosis have undergone whole-genome sequencing. The percentage of mutations shared by endometriosis and adjacent ovarian cancers ranged from $15 \%$ up to $98 \%$. PIK3CA and ARIDIA were present in all specimens of endometriosis synchronous with ovarian cancer [5]. It is worth mentioning that cancer development may be promoted by changes in miRNAs, observed in women with endometriosis. As aforementioned, mutations in miRNAs are associated with apoptosis dysregulation and neovascularization, which are essential to carcinogenesis [17]. Thirdly, study conducted by Wang et al. and dedicated to women affected by endometrioid ovarian cancer revealed differences in the age of onset and menopausal status, according to the presence or absence of coexistent endometriosis. Endometriosis-related ovarian cancers were diagnosed in approximately 5 years younger women and more often in premenopausal status in comparison with women without endometriosis $(\mathrm{p}=0.028)$. Moreover, patients with endometriosis were more likely to be diagnosed with early-stage and grade 1 ovarian cancer $(\mathrm{p}<0.001)$. Authors speculated that the relation between endometriosis and cancer may be explained by local estrogen production in endometriotic tissue. Higher levels of estrogen may drive a growth of ovarian tumor. Other explanation assumed that decrease in estrogen levels in postmenopausal age may cause a reduction in the incidence of endometriosis in this group of women [25].

\section{Prevention of ovarian cancer in women with endometriosis}

Evident progress in the researches about potential malignant transformation of endometriosis may give rise to the creation of the area of prevention. Due to early identification of mutations, such as ARIDIA, PTEN, PIK3CA in endometrial tissue, it may be possible to select women with higher risk of ovarian cancer development [3]. Some studies suggested a creation of genomic classification of endometriosis that could provide more specific information about prognostic and predictive factors. The knowledge about the presence of mentioned mutations and other risk factors may ease the decision about proper medical approach [2].

Studies confirmed that long-term use of oral contraceptive (OCs) pills reduces the risk of ovarian cancer in women with endometriosis. 
The greatest benefit was achieved by women with ten years of OCs use [26]. Insofar as the treatment of endometriosis with the use of OCs is commonly known and its protective action may be treated as a "useful side effect", surgical methods are more controversial. Meta-analysis conducted by Wang et al. showed that tubal ligation in women with endometriosis is associated with a major reduction in the risk of developing ovarian cancer. Tubal ligation is considered to be a mechanical barrier for retrograde menstruation. It supports the model of ovarian cancer originating from carcinous cells disseminated from the uterus. However, study confirmed no association between hysterectomy and epithelial ovarian cancer [27].

Average risk of developing ovarian cancer in women with benign endometriosis is not significantly higher than in the general population. In the general population it is one woman from 76, and in women in endometriosis- less than two women in 100. Therefore, in the group of women with benign endometriosis, invasive methods like bilateral salpingooophorectomy are not validated. This surgical intervention is associated with high risk of the aftermath of early menopause and also surgical complications [28]. For this purpose, genomic-based classification could enable the selection of women who should undergo that kind of surgical interventions, due to the assessing the benefits and harms [2].

\section{Summary}

Endometriosis remains not a fully known disease. However, it is one of the most frequent gynecological problems among women in reproductive age. Endometriosis is related with pelvic pain and infertility, likewise many studies deliver evidence for greater risk of developing a few types of ovarian cancer. Genetic testing - in order to confirm the presence or absence of some valid mutations - may be used for the settlement of a risk of ovarian cancer. The awareness of this risk could expedite decisions about proper medical approach. Further studies about the association between endometriosis and ovarian tumors are necessary to understand the molecular biology of both of them. One day this knowledge may affect the reduction in the incidence and mortality of ERONs.

\section{References}

[1] Králíčková M, Losan P, Vetvicka V. Endometriosis and cancer. Womens Health (Lond). 2014; 10(6): 591-7. doi: 10.2217/whe.14.43.

[2] Anglesio MS, Yong PJ. Endometriosis associated Ovarian Cancers. Clin Obstet Gynecol. 2017; 60(4): 711-727. doi: 10.1097/GRF.0000000000000320.

[3] Garavaglia E, Sigismondi C, Ferrari S, Candiani M4. The origin of endometriosisassociated ovarian cancer from uterine neoplastic lesions. Med Hypotheses. $2018 ; 110: 80-82$. doi: 10.1016/j.mehy.2017.11.006

[4] Pearce CL, Templeman C, Rossing MA, Lee A, Near AM, Webb PM, et al. Association between endometriosis and risk of histological subtypes of ovarian cancer: a pooled analysis of case-control studies. Lancet Oncol. 2012; 13(4): 385-394. doi: 10.1016/S14702045(11)70404-1

[5] Anglesio MS,i Bashashati A, Wang YK, Senz J, Ha G, Yang W, et al. Multifocal endometriotic lesions associated with cancer are clonal and carry a high mutation burden. $\mathrm{J}$ Pathol. 2015; 236(2): 201-209. doi: 10.1002/path.4516 
[6] Mehedintu C, Plotogea MN, Ionescu S, Antonovici M. Endometriosis still a challenge. J Med Life. 2014; 7(3): 349-357

[7] Yuan Z, Cragun J, Chambers S, Zheng W, Wang Y. Cell origin of endometriosis: contribution by the fallopian tube epithelium. Am J Clin Exp Obstet Gynecol. 2013; 1(1): 37-42 ISSN:2330-1899/AJCEOG1310002

[8] Shafrir AL, Farland LV, Shah DK, Harris HR, Kvaskoff M, Zondervan K, et al. Risk for and consequences of endometriosis: A critical epidemiologic review. Best Pract Res Clin Obstet Gynaecol. 2018; 51:1-15. doi: 10.1016/j.bpobgyn.2018.06.001

[9] Mori T, Ito F, Koshiba A, Kataoka H, Tanaka Y, Okimura H, et al. Aromatase as a target for treating endometriosis. J Obstet Gynaecol Res. 2018; 44(9): 1673-1681. doi: 10.1111/jog.13743.

[10] Basta P, Koper K, Kazmierczak W, Wisniewski M, Makarewicz A, Dutsch-Wicherek M, et al. The biological role of Treg cells in ectopic endometrium homeostasis. Histol Histopathol. 2014; 29(10):1217-33. doi: 10.14670/HH-29.1217

[11] Riccio LDGC, Santulli P, Marcellin L, Abrão MS, Batteux F, Chapron C. Immunology of endometriosis. Best Pract Res Clin Obstet Gynaecol. 2018; 50:39-49. doi: 10.1016/j.bpobgyn.2018.01.010

[12] Gogacz M, Winkler I, Bojarska-Junak A, Tabarkiewicz J, Semczuk A, Rechberger T, et al. Increased percentage of Th17 cells in peritoneal fluid is associated with severity of endometriosis. J Reprod Immunol. 2016; 117:39-44. doi: 10.1016/j.jri.2016.04.289

[13] Izumi G, Koga K, Takamura M, Makabe T, Satake E, Takeuchi A, et al. Involvement of immune cells in the pathogenesis of endometriosis. J Obstet Gynaecol Res. 2018; 44(2):191198. doi: $10.1111 /$ jog. 13559

[14] Thiruchelvam U, Wingfield M, O'Farrelly C. Natural Killer Cells: Key Players in Endometriosis. Am J Reprod Immunol. 2015; 74(4):291-301. doi: 10.1111/aji.12408

[15] Porpora MG, Scaramuzzino S, Sangiuliano C, Piacenti I, Bonanni V, Piccioni MG et al. High prevalence of autoimmune diseases in women with endometriosis: a case-control study. Gynecol Endocrinol. 2020 Apr;36(4):356-359. doi: 10.1080/09513590.2019.1655727

[16] Sapkota Y, Steinthorsdottir V, Morris AP, Fassbender A, Rahmioglu N, De Vivo I, et al. Meta-analysis Identifies Five Novel Loci Associated With Endometriosis Highlighting Key Genes Involved in Hormone Metabolism. Nat Commun. 2017; 24;8:15539. doi: 10.1038/ncomms 15539

[17] Gilabert-Estelles J, Braza-Boils A, Ramon LA, Zorio E, Medina P, Espana F, et al. Role of microRNAs in gynecological pathology. Curr Med Chem. 2012; 19(15) :2406-13. doi: $10.2174 / 092986712800269362$

[18] Maeda D, Shih IeM. Pathogenesis and the role of ARID1A mutation in endometriosisrelated ovarian neoplasms. Adv Anat Pathol. 2013; 20(1):45-52. doi: 10.1097/PAP.0b013e31827bc24d

[19] Alcázar JL, Guerriero S, Ajossa S, Parodo G, Piras B, Peiretti M, et al. Extragenital endometrial stromal sarcoma arising in endometriosis. Gynecol Obstet Invest. 2012;73(4):265-71. doi: 10.1159/000336522.

[20] Nezhat FR, Apostol R, Nezhat C, Pejovic T. New insights in the pathophysiology of ovarian cancer and implications for screening and prevention. Am J Obstet Gynecol. 2015; 213(3):262-7. doi: 10.1016/j.ajog.2015.03.044. 
[21] Ñiguez Sevilla I, Machado Linde F, Marín Sánchez MDP, Arense JJ, Torroba A, Nieto Díaz A, et al. Prognostic importance of atypical endometriosis with architectural hyperplasia versus cytologic atypia in endometriosis-associated ovarian cancer. J Gynecol Oncol. 2019; 30(4): e63. doi: 10.3802/jgo.2019.30.e63

[22] Mangili G, Bergamini A, Taccagni G, Gentile C, Panina P, Viganò P, et al. Unraveling the two entities of endometrioid ovarian cancer: a single center clinical experience. Gynecol Oncol. 2012; 126(3): 403-7. doi: 10.1016/j.ygyno.2012.05.007

[23] Falconer H, Sundqvist J, Xu H, Vodolazkaia A, Fassbender A, Kyama C, et al. Analysis of common variations in tumor-suppressor genes on chr1p36 among Caucasian women with endometriosis. Gynecol Oncol. 2012; 127(2): 398-402. doi: 10.1016/j.ygyno.2012.08.013

[24] Munksgaard PS, Blaakaer J.The association between endometriosis and ovarian cancer: a review of histological, genetic and molecular alterations. Gynecol Oncol. 2012 Jan;124(1):164-9. doi: 10.1016/j.ygyno.2011.10.001.

[25] Wang S, Qiu L, Lang JH, et al. Prognostic analysis of endometrioid epithelial ovarian cancer with or without endometriosis: a 12-year cohort study of Chinese patients. Am J Obstet Gynecol 2013 ;209: 241.e1-9 doi: 10.1016/j.ajog.2013.05.032

[26] Vercellini P, Viganò $\mathrm{P}$, Buggio L, Makieva S, Scarfone G, Cribiù FM, et al. Perimenopausal management of ovarian endometriosis and associated cancer risk: When is medical or surgical treatment indicated? Best Pract Res Clin Obstet Gynaecol. 2018; 51:151168. doi: 10.1016/j.bpobgyn.2018.01.017

[27] Wang C, Liang Z, Liu X, Zhang Q, Li S. The Association between Endometriosis, Tubal Ligation, Hysterectomy and Epithelial Ovarian Cancer: Meta-Analyses.Int J Environ Res Public Health. 2016; 14;13(11)

[28] Kvaskoff M, Horne AW, Missmer SA. Informing women with endometriosis about ovarian cancer risk. Lancet. 2017 Dec 2;390(10111):2433-2434. doi: 10.1016/S01406736(17)33049-0. 